\title{
Aplicação da técnica de Grupo Focal em pesquisa da Rede-SANS sobre as ações de alimentação e nutrição na atenção básica em saúde
}

\author{
Application of the focus group technique in research with the SANS \\ network on procedures in food and nutrition in basic health care
}

\author{
Carla Maria Vieira1, Laís Sartori Santiago², Patrícia Cristina Wesolowski Tavares², \\ Adriana Brandt ${ }^{3}$, Flavia Negri ${ }^{4}$, Maria Rita Marques de Oliveira ${ }^{5}$
}

\begin{abstract}
Resumo
Grupo focal como recurso da pesquisa qualitativa é importante ferramenta na avaliação propositiva de projetos de articulação de redes e movimentos sociais. Seis grupos focais foram aplicados como uma das etapas de pesquisa do Projeto Rede de Segurança Alimentar e Nutricional Sustentável (Rede-SANS), rede social de articulação da academia com os movimentos populares e poder público, em defesa do Direito Humano à Alimentação Adequada. Este relato teve como objetivo discutir a experiência da aplicação da referida técnica para investigar as percepções de profissionais de saúde sobre as ações de alimentação e nutrição na Atenção Básica. Os grupos focais permitiram aproximação e troca de experiências de 52 profissionais de 13 municípios de diferentes regiões do Estado de São Paulo para debater questões de segurança alimentar no contexto da área da saúde e promoveu reflexões sobre a alimentação, vigilância nutricional e intersetorialidade, como temas emergentes nos grupos. A técnica aplicada se demonstrou adequada para investigar o tema em profundidade, em pouco tempo, com um número grande de profissionais de distintos contextos para além dos objetivos da pesquisa. A realização dos grupos focais possibilitou o fortalecimento do processo de articulação de rede para a promoção de ações locais de segurança alimentar e nutricional sustentável.

Palavras-chave: grupos focais; atenção primária à saúde; segurança alimentar e nutricional; programas e políticas de nutrição e alimentação.
\end{abstract}

\begin{abstract}
Focus groups are seen as important tools in qualitative research for evaluating projects aimed at articulating social networks and movements. Six focus groups were held as one of the stages in research as part of the Food Safety and Sustainable Nutrition Project (SANS). This social network articulates the academic world with social movements and government in defense of the human right to suitable nutrition. This report is aimed at discussing the experience of applying focus groups to the investigation of perceptions by health professionals of activities related to food and nutrition in basic health care. These groups made it possible to bring together 52 professionals from 13 municipalities in the State of São Paulo, Brazil, and they exchanged experiences and debated issues related to food safety in the area of health care. The group discussions identified
\end{abstract}

Trabalho realizado na Universidade Estadual Paulista "Júlio de Mesquita Filho" (UNESP) - Botucatu (SP), Brasil.

'Nutricionista, Doutora em Ciências Médicas/Saúde Mental pela Faculdade de Ciências Médicas da Universidade Estadual de Campinas (UNICAMP) -

Campinas (SP), Brasil; Pesquisadora Colaboradora no Departamento de Psicologia Médica e Psiquiatria na UNICAMP - Campinas (SP), Brasil; Docente do Curso de Nutrição da Universidade Metodista de Piracicaba (UNIMEP) - Piracicaba (SP), Brasil.

${ }^{2}$ Nutricionista pelo Curso de Nutrição da UNIMEP - Piracicaba (SP), Brasil; Bolsista do Conselho Nacional de Desenvolvimento Científico e Tecnológico (CNPq) no Instituto de Biociências da UNESP - Botucatu (SP), Brasil.

${ }^{3}$ Graduada em Direito pela Universidade Federal de Mato Grosso - Cuiabá (MT), Brasil; Membro do Instituto Harpia Harpyia; Bolsista do CNPq no Instituto de Biociências pela UNESP - Botucatu (SP), Brasil.

${ }^{4}$ Mestranda na Faculdade de Saúde Pública da Universidade de São Paulo (USP) - São Paulo (SP), Brasil; Bolsista do CNPq no Instituto de Biociências pela UNESP - Botucatu (SP), Brasil.

${ }^{5}$ Doutora em Ciências dos Alimentos pela Faculdade de Farmácia da USP - São Paulo (SP), Brasil; Docente no Curso de Nutricão da UNESP - Botucatu (SP), Brasil. Endereço para correspondência: Carla Maria Vieira - Rua Petrópolis, 219/315 - CEP:13104-150 - Campinas (SP), Brasil - E-mail: cmvieira@unimep.com.br Fonte de financiamento: FINEP Convênio: 01.10.0466.00.

Conflito de interesses: nada a declarar. 
eating, nutrition vigilance and intersectoriality as emerging topics. The focus group technique proved to be a suitable tool for investigating the topic quickly and in depth, with a large number of professionals working in different contexts. In fact, the discussions went beyond the objectives of the research, since the group work made it possible to strengthen the process of articulation carried out by a network that promoting local measures in food safety and sustainable nutrition.

Keywords: focal groups; primary health care; food and nutrition security; nutrition programs and policies.

\section{INTRODUÇÃO}

A técnica de Grupo Focal (GF) é uma das diversas modalidades de entrevista grupais e/ou grupo de discussão ${ }^{1}$. Diferente de uma entrevista individual, o GF não está restrito simplesmente à alternância de perguntas de um pesquisador e respostas dos participantes ${ }^{2}$. Ele está entre as técnicas de abordagem rápida, utilizadas pelos epidemiologistas e educadores de saúde, permitindo conhecer, de forma eficaz, ligeira e com baixo custo, características psicológicas, sociológicas e culturais de determinados grupos da população e dos processos dinâmicos de interação entre eles².

Estruturado primeiramente por Merton e Kendall durante a década de 1940, o GF foi utilizado em pesquisas sociais com soldados durante a Segunda Guerra Mundial ${ }^{3}$. Neste período, a aplicação da técnica de GF teve como objetivo conhecer a eficácia do material de treinamento para as tropas e o efeito de propagandas persuasivas ${ }^{4,5}$.

Portanto, é sabido que esta técnica foi inicialmente utilizada fora do campo de pesquisa. Lazarsfeld et al. são citados como responsáveis por adaptar o GF para ser aplicado em pesquisas de mercado ${ }^{3}$. Somente na década de 1950, Thompson e Demerath ${ }^{3}$ utilizaram o GF para estudar fatores que influenciavam a produtividade de grupos de trabalho.

De acordo com Lervolino e Pelicioni ${ }^{5}$, por volta de 30 anos depois destas primeiras experiências, no início dos anos 1980, a técnica do GF foi utilizada em estudos na área de saúde, com os primeiros trabalhos publicados em 1984. Na década de 1990, foi expressivo o aumento de pesquisas utilizando esta técnica ${ }^{5}$. No Brasil, a socióloga Márcia Wesphtal ${ }^{2,6}$ é reconhecida como a pesquisadora responsável por utilizar a técnica de GF na área de saúde e associar a pesquisa qualitativa ao trabalho com movimentos sociais.

Atualmente, esta técnica de pesquisa tem sido utilizada por vários profissionais no desenvolvimento de pesquisas em educação em saúde com diferentes propósitos. Dentre eles, o de avaliar serviços ou intervenções de material institucional, para o planejamento e acompanhamento de programas, como instrumento de avaliação formativa, implementação e avaliação de programas, entre outras aplicações ${ }^{1}$. A aplicação da técnica possibilita a obtenção de dados com certo nível de aprofundamento em um curto período de tempo, devido à exposição de diferentes opiniões pessoais dos participantes sobre o mesmo assunto ${ }^{6}$.

A técnica de GF é uma importante ferramenta de pesquisa quando visa à avaliação propositiva de projetos que envolvem a organização de redes de articulação e movimentos sociais. No entanto, muitos estudos publicados que referem a sua aplicação apresentam escassas informações sobre os mecanismos utilizados, dificuldades e características específicas desta técnica que devem ser levados em consideração no momento da escolha do instrumento de coleta de dados do projeto de investigação ${ }^{6}$.

Pesquisas que se vinculam ao trabalho de movimentos sociais tem carência de espaços para o debate entre pesquisadores e ativistas da sociedade civil ${ }^{7}$. Nesse sentido, a descrição desta experiência poderá ser útil para aqueles que estão envolvidos, tanto na produção de conhecimentos em saúde coletiva, quanto para outros que se reconhecem envolvidos em movimentos sociais no campo da segurança alimentar.

Este artigo apresenta-se, portanto, com a proposta de discutir questões metodológicas na aplicação da técnica de GF para atender os objetivos de um projeto de pesquisa sobre as ações de alimentação e nutrição na Atenção Básica (AB). Tal projeto de pesquisa se insere em um projeto mais amplo que se articula enquanto movimento social de articulação de uma rede de defesa e promoção da alimentação saudável, adequada e solidária, o projeto de implantação da Rede de Defesa e Promoção da Alimentação Saudável Adequada e Solidária (Rede-SANS) ${ }^{8}$.

A descrição do percurso metodológico da pesquisa em que foram inseridos os GF é apresentada na seção Métodos. Na seção seguinte, Resultados e Discussão, buscamos apresentar os dados referentes à aplicação da técnica discutidas em quatro subsessões, como categorias de análise que emergiram no processo de discussão da aplicação da técnica de GF. As questões emergentes foram: Acolhimento e interação dos participantes; Características da equipe de pesquisa; Questões práticas que viabilizam a aplicação da técnica; Para além da técnica de pesquisa.

\section{MÉTODO}

A técnica de GF foi aplicada em uma das etapas da pesquisa inserida no projeto Rede-SANS ${ }^{9,8}$. A Rede-SANS 
é uma rede social em implantação desde 2009 que tem em vista a articulação da academia com os movimentos populares e o poder público, em defesa do Direito Humano à Alimentação Adequada (DHAA) e à Saúde ${ }^{8}$. As atividades da articulação de redes foram sistematizadas a partir do projeto "Rede de Municípios Promotores da Segurança Alimentar Nutricional Sustentável" subsidiado pelo Ministério da Ciência, Tecnologia e Inovação/Financiadora de Estudos e Projetos (FINEP), executado pela Fundação do Instituto de Biociências de Botucatu (Universidade Estadual Paulista "Júlio de Mesquita Filho" - UNESP), tendo como coexecutor o Instituto Harpia Harpyia ${ }^{8}$.

A Rede-SANS conta com as ações de Articuladores Locais (AL) e Articuladores Regionais (AR) nos municípios do Estado de São Paulo. Os articuladores estão voltados à integração, produção e difusão de conhecimentos e tecnologias para o monitoramento nutricional e promoção da alimentação adequada, saudável e solidária, nas diferentes comunidades. A articulação foi iniciada com 27 municípios paulistas e atualmente integra 29 municípios ${ }^{8}$.

O projeto de pesquisa, inserido no projeto de articulação da Rede-SANS, teve seu início em 2011 e o objetivo foi elaborar um diagnóstico propositivo quanto à atuação do Sistema de Vigilância Alimentar e Nutricional (SISVAN) no Estado de São Paulo. Os pesquisadores delinearam o estudo considerando a oferta de serviço de orientação e educação nutricional para populações atendidas nas Unidades Básicas de Saúde (UBS) assim como, os recursos do munícipio e da unidade para realização das atividades de monitoramento e promoção da alimentação saudável adequada e solidária e também, os procedimentos da equipe de saúde para obtenção de dados antropométricos nas unidades de saúde 8 . O projeto de pesquisa foi aprovado pelo Comitê de Ética em Pesquisa da Universidade Estadual Paulista, Faculdade de Medicina de Botucatu sob o protocolo CEP 3728-2010 em 08 de novembro de 2010.

A primeira fase da pesquisa, estudo transversal, de caráter quantitativo, foi realizada em 65 municípios do Estado de São Paulo. A escolha dos municípios ocorreu de forma aleatória, porém, foi preservada a escolha de municípios representativos das seis regiões do Estado que a Rede-SANS vem atuando. As seis regiões consideradas foram delimitadas pela definição de territórios da cidadania, proposto pelo governo federal ${ }^{8,10}$.

Para o desenvolvimento deste estudo qualitativo, que teve como missão aprofundar o conhecimento a respeito das ações de alimentação e nutrição na $\mathrm{AB}$, no Estado de São Paulo, anteriormente investigadas com instrumentos quantitativos, optou-se pela aplicação da técnica de GF.

A escolha pela técnica de GF ocorreu em função de duas questões centrais: a primeira questão diz respeito à amplitude territorial da pesquisa e de seu universo amostral. Para reunir um conjunto de informações de cunho qualitativo sobre a vivência de profissionais da $\mathrm{AB}$ de regiões e municípios tão diversos a aplicação de GF apresentava grandes possibilidades de êxito. O segundo elemento, que colaborou com a decisão pela técnica aplicada, foi à disponibilidade de condições financeiras e tecnológicas do projeto e a composição da equipe de pesquisadores, com experiência prévia consolidada no campo da pesquisa qualitativa em saúde, e particularmente com a condução de grupos focais.

Esta técnica demonstrou a vantagem de envolver vários atores sociais, oriundos de 13 municípios paulistas (Quadro 1), já conhecidos pelos AL e AR da Rede-SANS e alcançar um nível de aprofundamento satisfatório, a partir do debate estabelecido em torno das questões de SAN na $\mathrm{AB}$.

A delimitação do universo amostral ocorreu em reunião ordinária da coordenação da Rede-SANS e Articulares Regionais. Mantido o critério de divisão por região foi utilizado o seguinte critério para eleição dos municípios que compuseram a amostra: um município já pesquisado (fase quantitativa) e também participante da Rede-SANS e um município somente pesquisado, porém, não participante da Rede .

$\mathrm{Na}$ amostra selecionada, houve a preocupação de manter a representação de municípios de pequeno, médio e grande

Quadro 1. Composição dos grupos focais de acordo com as regiões do Estado de São Paulo, municípios representados e número de profissionais que participaram dos encontros Botucatu, 2012

\begin{tabular}{|lccc|}
\hline Grupo & Região & Municípios representados & $\begin{array}{c}\text { Número de } \\
\text { profissionais }\end{array}$ \\
1 & Presidente Prudente & Martinópolis, Pirapozinho, Presidente Prudente e Presidente Venceslau & 11 \\
2 & Ribeirão Preto & Ribeirão Preto & 8 \\
3 & São José do Rio Preto & Cedral, Mirassol e São José do Rio Preto & 10 \\
4 & Marília & Marília e Pompéia & 11 \\
5 & Itapeva & Itapeva e Nova Campina & 6 \\
6 & Registro & Registro & 6 \\
\hline
\end{tabular}


Tabela 1. Características sociodemográficas dos participantes

$\begin{array}{lc}\text { Características dos participantes } & \text { Total } \\ \text { Sexo } & \\ \text { Feminino } & 49 \\ \text { Masculino } & 03 \\ \text { Faixa etária } & \\ \geq 20-30 & 18 \\ >30-50 & 30 \\ \geq 50 & 04 \\ \text { Anos de escolaridade } & \\ 11 \text { anos } & 13 \\ \text { Mais que } 11 \text { anos } & 39 \\ \text { Tempo de atuação na AB } & \\ \text { <1 ano } & 06 \\ >1 \text { ano até } 5 \text { anos } & 20 \\ \text { Entre 5 a } 10 \text { anos } & 10 \\ \text { Mais que } 10 \text { anos } & 16 \\ \text { Profissão dos participantes } & \\ \text { Agente comunitário em saúde } & 17 \\ \text { Enfermeiro } & 17 \\ \text { Auxiliar/técnico de enfermagem } & 10 \\ \text { Nutricionista } & 04 \\ \text { Médico } & 01 \\ \text { Farmacêutico } & 01 \\ \text { Odontólogo } & 01 \\ \text { Auxiliar de saúde bucal } & 52 \\ \text { Total } & \end{array}$

porte, mas esta regra não chegou a ser determinante para participação nos GF. A participação estava principalmente condicionada aos municípios serem capazes de aderir à proposta de formar grupos com pessoas interessadas em debater o tema sugerido.

Os AR e AL fizeram os convites para os profissionais e estes aceitaram participar voluntariamente do GF realizado em sua região. Os diálogos foram gravados após o consentimento dos participantes e assinatura do Termo de Consentimento Livre e Esclarecido.

$\mathrm{Na}$ aplicação de GF, para a experiência aqui analisada, os sujeitos selecionados para o estudo eram profissionais de saúde da $\mathrm{AB}$. Os GF foram formados com média de dez participantes, com faixa etária entre 21 e 40 anos, maioria do sexo feminino, com mais de 11 anos de estudo, média de 1 a 5 anos de atuação na $\mathrm{AB}$ e com maior número de profissionais de enfermagem e agentes comunitários de saúde (Tabela 1).

Durante as entrevistas dos GF foi utilizado um roteiro de entrevista, o qual inclui um número pequeno de questões, aproximadamente 5 ou 6 , contendo temas chave a serem investigados e que apoiaram a discussão. De acordo com as referências metodológicas ${ }^{1,2}$, este roteiro deve ser formulado em função dos objetivos da pesquisa e pré-testado em uma audiência semelhante a que participará do estudo.
Seguindo as recomendações da literatura ${ }^{6,11}$ para a coleta de dados, foi aplicado um roteiro elaborado com 5 questões que envolviam 3 temas, todos relacionados à atuação dos profissionais da $\mathrm{AB}$ no campo da alimentação e nutrição. Este roteiro foi previamente testado com um grupo de profissionais de um município participante da Rede-SANS, com participantes selecionados pelo AL. Essa experiência preliminar possibilitou realizar adequações no roteiro e, também, verificar que sua aplicação era potencialmente eficaz para conduzir a discussão dos próximos grupos e atingir os objetivos da pesquisa.

\section{RESULTADOS E DISCUSSÃO}

\section{Acolhimento e interação dos participantes}

A recepção dos participantes é um momento relevante e não deve ser banalizado, pois durante este período, os participantes estão apreensivos e retraídos sobre o que ocorrerá durante o $\mathrm{GF}^{11}$. Uma recepção atenciosa favorece a interação entre os participantes e a equipe de pesquisa.

A aplicação de dinâmicas de grupo após a recepção e para o início das atividades, como recomendada na literatura ${ }^{4,11}$, favorece a desinibição e descontração dos participantes e da equipe de pesquisa. A prática de atividades lúdicas, de rápida e simples aplicação é capaz de proporcionar maior concentração e envolvimento no debate proposto.

A escolha e preparação de um espaço físico sem ruídos excessivos para garantir a gravação, mas ao mesmo tempo com condições adequadas de acomodação dos participantes, foram os primeiros recursos elegidos pelo grupo de pesquisa da Rede-SANS para a etapa de acolhimento e integração. $\mathrm{O}$ convite para uma prática de alongamento, quando o grupo estava completo, teve boa adesão dos participantes, ocupou em torno de 5 minutos e se demonstrou de fácil aplicação.

Para a etapa de apresentação, foi proposta a formação de duplas de participantes (com sugestão de escolha de alguém desconhecido) para o estabelecimento de um diálogo breve sobre um fato inusitado que havia ocorrido durante a semana. Após a apresentação dos fatos inusitados e das pessoas, era observado um ambiente de descontração e a interação dos participantes era alcançada, o que permitia dar início à explanação dos objetivos da pesquisa, o estabelecimento de limites de horário para finalização, assim como a solicitação de consentimento para gravação.

De acordo com a literatura ${ }^{1,11}$, existem limitações na aplicação da técnica de GF em relação a situações em que o pesquisador precisa proporcionar um ambiente favorável ao participante para compartilhar detalhes íntimos de seu 
cotidiano. Por isso, deve-se evitar a realização de GF onde os participantes não se sintam à vontade e que haja fortes discordâncias de opinião ${ }^{1}$.

A flexibilidade na aplicação da técnica de GF é salientada por Wesphtal ${ }^{2}$ com incorporação de dinâmicas de grupo que podem permitir aos pesquisadores a percepção sobre os processos de interação entre os participantes e a identificação de resistências individuais ${ }^{12}$. As questões subjetivas que interferem na dinâmica grupal demandam a aplicação de recursos lúdicos e interativos para a obtenção de um ambiente favorável, com menor constrangimento possível ${ }^{1,13}$.

A aplicação da técnica de dinâmica de grupo com objetivo de propiciar um ambiente favorável à exposição das opiniões, sentimentos e relatos durante o desenvolvimento dos grupos focais $^{1,11}$ possibilitou que os participantes compartilhassem experiências pessoais e profissionais relativas ao comportamento alimentar e práticas alimentares.

Dificuldades em relação às mudanças de hábitos alimentares, perdas e processos de adoecimento relacionados à alimentação foram expostos pelos sujeitos do estudo. Ou seja, a dinâmica dos grupos se configurou, na maior parte dos encontros, com suficiente estrutura para acolher relatos da intimidade de prática profissional e das relações familiares, mediadas pelos alimentos e pela comensalidade.

\section{Características da equipe de pesquisa}

Para garantir a aplicação dos GF como técnica de pesquisa qualitativa de forma a preservar o rigor científico é necessário contar com uma equipe de pesquisadores de pelo menos 3 integrantes e idealmente com $5^{11}$. A partir da nossa experiência percebemos que com a equipe completa, há melhor condição de coleta das informações e melhores chances de aprofundamento dos debates e cruzamento das narrativas com as expressões não verbais no processo de análise dos dados. Ou seja, a equipe com no mínimo 4 e idealmente 5 integrantes proporciona maior qualificação dos dados obtidos.

A habilidade exigida da equipe de pesquisadores para aplicação da técnica de GF perpassa também pela capacidade de condução das discussões para evitar o desvio do tema proposto para o debate ${ }^{14}$. O moderador precisa, portanto, de certa prática de coordenação e condução de atividades com grupos para permitir a flexibilidade em relação aos depoimentos e expressão de opiniões e sentimentos dos participantes, garantir a atenção dos mesmos, estimular que todos participem e inibir polarizações ${ }^{14}$. Além disso, o moderador tem que ser capaz de reconduzir o debate do tema sempre que o grupo desviar do assunto. Formular conclusões preliminares para fazer a validação das sínteses provisórias com o próprio grupo também é uma tarefa fundamental do moderador para que o objetivo do GF seja atingido, com obtenção dos dados de qualidade ${ }^{2,6,11}$.

O registro das falas em texto por 1 ou 2 pesquisadores é recomendado pela literatura, como uma maneira de garantir que os dados não sejam perdidos, caso ocorra algum problema na gravação ${ }^{11}$. No entanto, o referido registro quando digitalizado por pesquisadores com boa capacidade de digitação e envolvimento no tema, produz um texto de ótima qualidade e pode ser utilizado para análise dos dados após a complementação de algumas lacunas identificadas na gravação. Essa estratégia pode substituir o trabalho de transcrição de toda a gravação, demonstrando adequação para pesquisas que demandam a realização de muitos grupos, envolvendo um grande número de informações.

Ainda sobre as características da equipe, salientamos que as observações das expressões não verbais devem ser registradas durante a realização dos grupos, por um pesquisador que se prepara para tal tarefa. O preparo do pesquisador diz respeito ao treino em captar e registrar manifestações corporais que podem expressar dados que não estão ditas nas expressões verbais, sobre as questões discutidas. Não se trata de registrar todo e qualquer movimento corporal dos participantes, mas sim aqueles movimentos que confirmam ou expressam desacordo em relação a determinado depoimento. O significado do silêncio, das expressões de desinteresse, cansaço, desatenção, tensão, entre outros devem ser interpretados pelo pesquisador designado para a observação e registro das expressões não verbais. As expressões não verbais são importantes para a pesquisa pois complementam a compreensão dos conteúdos latentes do discurso, na etapa de análise dos dados ${ }^{6,11}$.

Vale ressaltar ainda que a equipe de pesquisa deste estudo foi formada por profissionais envolvidos em movimentos sociais no campo da segurança alimentar e nutricional e por integrantes do projeto de articulação da Rede-SANS. Essa duplicidade de papéis dos pesquisadores foi positiva, pois a formação técnica, o envolvimento e a atualização do grupo no assunto permitiram a formulação de perguntas e sínteses pertinentes.

Em outro sentido, a identificação dos pesquisadores como integrantes da Rede-SANS pode ter inibido algumas críticas e opiniões de alguns participantes sobre as atividades desenvolvidas pela Rede-SANS no município ou na unidade de saúde dos participantes. A partir da aplicação preliminar do roteiro, foi possível perceber a interferência do envolvimento dos pesquisadores, no contexto de implantação da Rede-SANS, na coleta de dados, fazendo com que o grupo de pesquisa evitasse manifestações de apoio e divulgação do referido projeto durante a realização do GF. 
A formação de uma equipe de pesquisadores com domínio da técnica de GF e também com conhecimento sobre o assunto a ser pesquisado, porém, sem envolvimento direto com o contexto em que se desenvolve o projeto ou o movimento social em que se insere o projeto de pesquisa, poderia evitar a inibição e constrangimento dos participantes no debate sobre o movimento social. Nesse sentido, alguns autores ${ }^{3,15}$ recomendam certo distanciamento dos pesquisadores em relação ao movimento social.

\section{Questões práticas que viabilizaram a aplicação da técnica}

Os equipamentos a serem utilizados para digitação e gravação durante o GF devem estar sempre disponíveis e em duplicata para garantir o registro dos dados ${ }^{11}$. A partir da vivência com o GF, verificamos que é necessário ter a disponibilidade de equipamentos de gravação eficientes e em bom estado de manutenção para obter um registro de qualidade.

O número de sujeitos estudados com a aplicação da técnica do GF foi pequeno se comparado com o estudo descritivo amostral desenvolvido na primeira fase da pesquisa ${ }^{6}$. Contudo, as referências consultadas indicam que a amostra pequena é necessária para que seja possível se debruçar e conhecer a subjetividade dos participantes, na busca pela contextualização e interpretação dos dados obtidos através dos debates e trocas de experiências de vida²

O GF é composto normalmente de 6 a 10 participantes não familiares uns aos outros, os quais devem apresentar pelo menos um traço comum, determinado pelo objetivo do estudo, como idade, sexo, nível de escolaridade, ocupação, entre outros $^{5,6}$. Os sujeitos são selecionados a partir de características em comum associadas ao tema que está sendo pesquisado ${ }^{6}$. Ressaltamos que os participantes selecionados para os GF possuíam um traço comum: atuantes da $\mathrm{AB}$, característica relevante para este estudo (Tabela 1).

A aplicação das sessões de GF teve em média a duração de uma hora e meia, como recomendado pela literatura ${ }^{6,16}$. Verificamos que após o tempo recomendado os participantes demonstravam cansaço e dispersão em relação aos assuntos discutidos.

Uma das limitações encontradas para aplicação da técnica é que normalmente conta com a boa vontade e a disponibilidade dos sujeitos para formar grupos em data e locais muitas vezes pouco convenientes para os mesmos². Com a experiência do GF, percebemos que a convocação dos participantes deve ser realizada com duas semanas de antecedência para que os convocados se organizem a fim de poder comparecer na data e horário marcado e também evitar o esquecimento. Um detalhe importante nesta etapa é que os participantes sejam avisados para chegar meia hora antes do início do encontro, pois o atraso atrapalha a condução e o horário do término do GF.
Após a finalização do GF, os participantes foram convidados a desfrutar de um lanche. Esses momentos finais, permeados pela comensalidade, permitiram que alguns participantes complementassem algumas ideias sobre as questões discutidas no grupo, de maneira mais descontraída.

Esses depoimentos e relatos feitos na informalidade foram registrados em diário de campo pelos pesquisadores, após a saída de todos os participantes. Reunidos por volta de 15 minutos, a equipe realizava uma breve reunião, após cada GF para identificação dos pontos mais importantes que emergiram nas discussões. Essa etapa final foi útil também para avaliar a organização e interação entre os membros da equipe de pesquisa para aperfeiçoamento da utilização da técnica.

\section{Para além da técnica de pesquisa}

A principal característica desta técnica é trabalhar com a "fala" dos participantes, os quais representam os sujeitos do estudo, permitindo que eles expressem suas percepções, crenças, valores, atitudes e representações sociais, e apresentem seus conceitos e opiniões sobre os temas discutidos ${ }^{2,6,11}$.

A vivência dos encontros para aplicação da técnica de GF permitiu confirmar que as pessoas precisam ouvir as opiniões dos outros antes de formar as suas próprias, e mudar de posição ou fundamentar melhor sua posição inicial quando expostas a discussão de grupo, conforme indicado na literatura $^{6,16}$. O moderador ao fazer as questões instiga os membros do grupo a pensarem e expressarem suas opiniões sobre o assunto. Quando o primeiro integrante faz sua colocação, sendo algo comum ou divergente ao grupo, os outros participantes podem se sentir motivados a fazer suas colocações e alimentar o debate e com isso viabilizar a coleta de dados, de acordo com os objetivos da pesquisa ${ }^{16}$.

Verificamos, contudo, que o debate permitiu, além da reflexão e expressão de opiniões, a troca de experiências relacionadas à prática de vigilância nutricional realizadas nas unidades de saúde, assim como a aproximação entre os profissionais para possíveis parcerias diante das dificuldades cotidianas no campo da alimentação e nutrição, as quais demandam ações intersetoriais (Quadro 2).

Portanto, para o grupo de pesquisa da Rede-SANS, a aplicação da técnica do GF foi além da coleta de dados. A interação de profissionais da $\mathrm{AB}$ de diferentes unidades de saúde de distintos municípios do Estado de São Paulo proporcionou a aproximação entre os participantes e pesquisadores, como previamente identificado na literatura ${ }^{6,5}$. Nesse sentido, os encontros dos profissionais da $\mathrm{AB}$ para a realização dos GF permitiram a avaliação das ações de alimentação e nutrição na $\mathrm{AB}$ e levantamento de problemas, mas também contribuiu para o processo de articulação das atividades do projeto de constituição da Rede-SANS. 
Quadro 2. Depoimentos dos participantes sobre o desenvolvimento do grupo focal

\section{Depoimentos}

Imaginei que poderia ser uma palestra sobre nutrição, mas [...] a gente aprendeu e compartilhou [...] Nós não nos conhecemos, mas trabalhamos em uma unidade.

É para a gente pensar um pouco!

[...] gosto desta integração, do que acontece fora e saber que a realidade não é a mesma nas outras unidades.

Serviu para troca de experiência que é muito boa...

[...] muito produtivo [...] informações (que) eu não sabia!

[...] estes assuntos todos [...] foi muito bom, muito proveitoso!

\section{Grupo Focal}

2

3

4

5

6

\section{Região}

Ribeirão Preto

São José do Rio Preto

Marília

Itapeva

Registro

\section{CONSIDERAÇÕES FINAIS}

A aplicação da técnica do GF foi viável para obter dados qualitativos tais como opiniões, valores e sentimentos expressados pelos participantes sobre os temas propostos durante o debate. Contudo para alcançar um ambiente favorável e acolhedor sugerimos a incorporação de dinâmicas de grupo que permitiram melhor interação entre os participantes.

Dentre as características da equipe de pesquisadores para adequada aplicação da técnica de GF salientamos que a composição com 4 ou 5 integrantes permite a condução adequada dos grupos, gravação e digitação dos relatos, observação e registro das expressões não verbais para posterior cruzamento dos dados para análise. A preparação do moderador como coordenador de grupos é relevante no sentido de viabilizar uma boa dinâmica grupal.

A observação de questões práticas que viabilizem o desenvolvimento desta modalidade de pesquisa qualitativa é recomendada a fim de não comprometer o bom andamento da coleta de dados. Convites antecipados aos sujeitos da pesquisa, definição do local e horário para a realização dos encontros, assim como, a oferta de um lanche como retribuição a participação dos sujeitos, especialmente quando isso ocorre de forma voluntária, são detalhes importantes a serem levados em conta na etapa de planejamento.

Consideramos, finalmente, que o GF tem potencialidades que vão além da técnica de pesquisa de cunho qualitativo. $\mathrm{O}$ projeto de pesquisa ao encontrar-se inserido no contexto de movimentos sociais tem a possibilidade de apoiar a articulação entre os participantes no desenvolvimento de parcerias e na realização de novos projetos. Salientamos, contudo, que a coleta de dados sofre interferências significativas nas reflexões dos grupos quando os pesquisadores estão excessivamente envolvidos no contexto do movimento social em que a pesquisa se desenvolve.

\section{REFERÊNCIAS}

1. Borges CD, Santos MAD. Aplicações da técnica do grupo focal: fundamentos metodológicos potencialidades e limites. Rev SPAGESP. 2005;6(1):74-80.

2. Westphal MF. Participação popular e políticas municipais de saúde: o caso de Cotia e Vargem Grande Paulista [tese]. São Paulo (SP): Faculdade de Saúde Pública. Universidade do Estado de São Paulo; 1992.

3. Morgan DL. Focus groups as qualitative research. Qualitative research methods series. California: Sage Publications; 1997.

4. Antoni DC, Martins C, Ferronato MA, Simões A, Maurente V, Costa F, et al. Grupo focal: Método qualitativo de pesquisa com adolescentes em situação de risco. Arq Bras Psicol. 2001;53(2):38-53.

5. Lervolino AS, Pelicioni MCF. A utilização do grupo focal como metodologia qualitativa na promoção da saúde. Rev Esc Enferm USP. 2001;35(2):115-21.

6. Westphal MF, Bógus CM, Faria MM. Grupos focais: experiências precursoras em programas educativos em saúde no Brasil. Bol Oficina Sanit Panam. 1996;120(6):472-82.

7. Silva MK. De volta aos movimentos sociais? Reflexões a partir da literatura brasileira recente. Rev Ciênc Soc Unisinos. 2010;46(1):2-9.

8. Fundação do Instituto de Biociências, Universidade Estadual Paulista. Rede de municípios promotores da segurança alimentar nutricional sustentável-Rede-SANS. Projeto de pesquisa e desenvolvimento; 2009.

9. Oliveira MRM. REDE-SANS-Rede defesa e promoção da alimentação saudável, adequada e solidária. In: World Nutrition Rio 2012. Rio de Janeiro; 2012. p. 1-14.
10. Brasil. Decreto n. ${ }^{\circ}$ 51.433, de 28 de dezembro de 2006. Cria unidade na Coordenadoria de Regiões de Saúde, da Secretaria da Saúde, altera a denominação e dispõe sobre a reorganização das Direções Regionais de Saúde e dá providências correlatas [Internet]. Diário Oficial [do] Estado de São Paulo. 2006 dez. 29 [acesso em 2012 nov 14]. Disponível em: $\mathrm{ftp} / / / \mathrm{ftp}$. saude.sp.gov.br/ftpsessp/bibliote/ informe_eletronico/2006/iels. dezembro.06/iels247/E_DC-51433_281206.pdf

11. Neto OC, Moreira MR, Sucena LFM. Grupos focais e Pesquisa Social Qualitativa: o debate orientando como técnica de investigação. In: XIII Encontro da Associação Brasileira de Estudos Populacionais. Ouro Preto; 2012. p. 1-26.

12. Afonso MLM. Oficinas em dinâmica de grupo na área da saúde. São Paulo: Casa do Psicólogo; 2006.

13. Failde I. Manual do facilitador para dinâmicas em grupo. Campinas: Papirus; 2007.

14. Andalo CSDA. O papel do coordenador de grupos. Psicol USP. 2001; 12(1):135-152.

15. Gondrim SMG. Grupos focais como técnica de investigação qualitativa: desafios metodológicos. Paidéia. 2003;12(24):149-61.

16. Carlini-Cotrim B. Potencialidade da técnica qualitativa grupo focal em investigações sobre abuso de sustâncias. Rev Saúde Pública. 1996;30(3):285-93. 